\title{
Epiphrenic Diverticula. "Primum Non Nocere" (First Do Not Harm)
}

\author{
Giovanni Zaninotto \& Sheraz Markar \\ Department of Academic Surgery, Imperial College - St Mary's Hospital, London, UK
}

Corresponding author:

Giovanni Zaninotto, MD Senior Teaching Fellow

Honorary Consultant Department of Academic Surgery Imperial College - St Mary's Hospital E-mail: g.zaninotto@imperial.ac.uk
Epiphrenic diverticula (ED) are out-pouchings of the esophageal lumen most commonly originating in the distal third of the esophagus, close to the diaphragm. They have historically been considered 'pulsion'diverticula due to high intra-luminal pressures in a short segment of the esophagus in contrast with mid thoracic diverticula that are deemed to be caused by a chronic inflammatory processes starting from the mediastinal lymph nodes (due to granulomatous diseases, such as tuberculosis) $(1,2)$. The prevalence of ED is unknown:radiological studies (contrast esophagogram) have shown a prevalence of ED of around $0.015 \%$ in the US, $0.77 \%$ in Japan and $2 \%$ in Europe $(3,4)$. Trastek estimated the ratio of epiphrenic to Zenker's diverticula at 1:5 and, since the incidence of Zenker's diverticula is generally assumed to be less than 1/100,000/year, the estimated incidence of epiphrenic diverticula would be approximately 1/500,000/year; such a figure gives us an idea of just how rare diverticula of the thoracic esophagus are (5). Symptoms of ED are variable in type, severity and frequency: dysphagia, regurgitation, chest pain and /or aspiration problems are reported; most likely these symptoms are associate with the underlying motor disturbance rather than the ED itself (6), especially in case of small ED. The proportion of diverticula reportedly symptomatic enough to warrant surgery is highly variable, ranging from $0 \%$ to $50 \%(7,8)$.

Esophageal manometry (either perfused or high resolution manometry) reveals motor abnormalities in $75-90 \%$ of patients with ED (9). By using 24-hour prolonged manometry, Nehra demonstrated the presence of motility abnormalities in $100 \%$ of 21 patients investigated (10): the most common motor 
anomalies seen in these patients were achalasia and diffuse esophageal spasm (DES).

The clinical questions to address when dealing with ED are:

1. Do they always need treatment?

2. Which is the best approach? Open, minimally invasive or combined? Transthoracic, transabdominal or combined?

3. Should we always address an underlying esophageal motor disorder (even if undetected on manometry) or should we limit our intervention to diverticulectomy?

Given the rarity of the disease only cohort studies or case series have been published and most of the recommendations have a low grade of evidence.

\section{Do All Epiphrenic Diverticula Always Need Treatment?}

This is probably one of the most intriguing questions regarding ED. The natural history of epiphrenic diverticula is not clear and whether to operate or not has been amatter of debate for a long time. Orringer has often been quoted, 'A masterful inactivity in asymptomatic or mildly disturbing diverticula is a good practice even if, in this time of minimally-invasive surgery and stapling devices, an esophageal diverticulectomy may representa tempting trophy for a hyperactive surgeon' (11).

The presence of a diverticulum cannot, per se, be considered an indication for surgery. The surgeon should balance the surgeryrelated risks against the potential benefits, carefully assessing the patient's symptoms and the risk of complications related to the presence of the diverticulum.

In general, severe dysphagia, regurgitation and contrast retention on esophagography, with an implicit or explicit risk of aspiration pneumonia, would be indications for surgical treatment. Although the diverticulum's size is not strictly related to the severity of patient's symptoms, the small diverticula especially if located proximally are often mildly symptomatic or asymptomatic and rarely require surgery, while large diverticula are often associated with food retention and regurgita- tion, more commonly need diverticulectomy.

The most severe complications related to a diverticulum in the thoracic esophagus are perforation or rupture of the diverticulum in the mediastinum, or (less frequently) progression to carcinoma within the diverticulum $(12,13)$. Aside from anecdotal reports from Altorki and Skinner (who strongly advocated surgery for all epiphrenic diverticula) with a high rate of aspiration (9/20 patients), and even a case of tracheo-esophageal fistula, the natural history of asymptomatic or mildly symptomatic diverticulum is difficult to predict (12), it has been estimated that symptoms develop or worsen in less than $10 \%$ of such patients $(14,15,16)$. On the other hand, in cases of epiphrenic diverticula that are moderately-to-severely symptomatic, the disease usually tends to progress (12). When evaluating a patient with ED the risk related to its surgical treatment should be balanced against the benefit of the operation. The overall mortality rate for surgery for epiphrenic diverticula is nearly 5\% (higher than in esophagectomy for "benign" diseases) and the morbidity rate is nearly $20 \%$, the most common complication being leakage (10, 17, $18,19,20,21)$ from the suture line.

Overall, in our experience, asymptomatic patients with small, incidentally diagnosed diverticula and mildly symptomatic cases with medium-sized pouches can be managed conservatively without any specific therapeutic intervention, apart from proton pump inhibitors (PPI) or H2-blockers, in the case of symptoms related to reflux or gastritis or addressing the underlying motor disorder. The non-surgical alternative of endoscopic pneumatic dilation proved valuable in symptomatic patients unfit for, or unwilling to undergo surgery with associated achalasia or hypertensive LES: they all benefited from this treatment and were symptom-free at 2-year follow-up at least (15).

If we compare the surgical complications with the fate of un-operated patients - in the medium term at least - then surgery can only be justified when patients suffer from severe, incapacitating symptoms (e.g. dysphagia, regurgitation and aspiration) and/or in case of 
existing or impending complications. Patients with minimal or no symptoms should be managed conservatively.

\section{Which is the Best Approach? Transthoracic, Transabdominal or Combined? Open, Minimally Invasive or Combined?}

Minimal access surgery has become popular for treating several benign esophageal diseases, including $\mathrm{ED}$. The first minimally-invasive approach used to treat epiphrenic diverticula was thoracoscopy - a logical evolution of the traditional thoracotomy access. The first report came from the Milan group, which treated 8 patients: the operation was completed thoracoscopically in 6, and there was one leak (22). A Dutch team reported on their experience with 5 patients operated using this method, with no mortality and one leak (23).

The introduction of linear flexible endostaplers capable of transecting the neck of the diverticulum while remaining as much as possible parallel to the esophageal axis has contributed to the diffusion of the laparoscopic approach. The trans-abdominal approach offers the advantage of an optimal view of the esophago-gastric junction when performing the myotomy. The feasibility of using the transabdominal route may be limited, however, by the distance of the diverticulum from the hiatus, inflammation and adhesions between the wall of the diverticulum and the mediastinal pleura and the size of the ED neck. Dissecting the proximal part of the diverticulum is more difficult when it is far from the hiatus and especially if there are adhesions to the pleura, with the likelihood of pleural tearing or laceration of the diverticulum wall.

Another problem to consider when choosing the laparoscopic approach is the size of the diverticulum neck: when it is very wide, it may take two or more firings of the endostapler to transect it and the crossing of the two sutures is often a potential site of leakage.

As a consequence of these difficulties the probability of having a leak from the suture line is pretty high, slightly above $20 \%$, as pointed out by two recent systematic reviews $(24,25)$.
The above-mentioned disadvantages of the laparoscopic technique warranted a combined approach especially in case of very large ED by using a thoracotomic or a thoracoscopic approach, either in lateral or prone position, to transect the diverticulum and addressing the motor disorder from the laparoscopic approach. The advantages of this approach allows the positioning of the stapler parallel to the esophagus, a good vision of the proximal tip of the stapler encompassing completely the diverticulum neck and the potential for oversewing the stapled suture line with the lateral edges of the esophageal muscle. After the diverticulectomy is accomplished the patient is rotated in supine position and the myotomy of the gastro-esophagel junction and partial fundoplication to prevent reflux via a laparoscopic route are performed.

\section{Should We Always Address an Underlying Esophageal Motor Disorder?}

An abnormal lower esophageal sphincter (hypertensive or non relaxing) and/or abnormal peristalsis in the lower part of the esophagus, with simultaneous contractions, are detectable in the majority of patients with ED. These motor abnormalities create a high-pressure segment that contributes to the protrusion of the esophageal wall: the functional disorder may be treated by a myotomy, which should start in line with the neck of the diverticulum, on the opposite side to the diverticulum to avoid interfering with subsequent pouch resection and muscle closure. The importance of the myotomy as a fundamental part of the treatment for epiphrenic diverticula was clearly illustrated in a series of 21 patients published by the Mayo Clinic: patients treated with diverticulectomy alone suffered from higher leakage and recurrence rates (24\% and $19 \%$, respectively) than those who had diverticulectomy with a myotomy $(0 \%$ for both leakage and recurrence rates) (26). In a recent systematic review of 25 observational studies on 511 patients Chang et al reported a lower rate of anastomotic leak when a myotomy was added form $26.1 \%$ (without myotomy) to $12.4 \%$ 
(with myotomy) (24).

When a myotomy is performed, a partial fundoplication is recommended to avoid the risk of subsequent gastroesophageal reflux. Data in the medical literature on the prevalence of gastroesophageal reflux (GER) after surgery with or without fundoplication for epiphrenic diverticula are very scarce, but GER occurs in over $30 \%$ of achalasia patients when myotomy is performed without adding an anti-reflux procedure and we can infer that the same may occur when a myotomy is added in ED (27).

Surgeons dealing with ED patients have to carefully consider the balance between the risks and benefits of surgical treatment. In our opinion, a conservative treatment is justified for small, asymptomatic or mildly symptomatic diverticula that can be managed successfully with PPI or esophageal dilations. Severe dysphagia, regurgitation or aspiration symptoms warrant surgery. Very large diverticula may represent per se an indication for surgery, even if they are asymptomatic. A thorough preoperative morphological and functional work-up is mandatory for appropriate patient selection and comprehensive planning of the surgical procedure. In our experience, the laparoscopic transhiatal approach enables the best myotomy to be performed, with fundoplication and - in most cases - the diverticulectomy too, though an additional thoracotomy may be needed for diverticula with large necks or ED located well above the pulmonary vein.

\section{References}

1. Harrington SW The surgical treatment of pulsion diverticula of the thoracicesophagus. Ann Surg. 1949;129(5):606-18.

2. Cross FS Esophageal diverticula related neuromuscular problems. Ann Otol Rhinol Laryngol. 1968;77(5):914-26.

3. Wheeler D Diverticula of the foregut. Radiology 1947; 49:476-481.

4. Schima W, Schober E, Stacher G, Franz P, Uranitsch K, Pokieser P, et al. Association of midoesophageal diverticulawith oesophageal motor disorders. Videofluoroscopy and manometry. Acta Radiol. 1997;38(1):108-14.

5. Trastek VF, Payne WS. Esophageal diverticula. În: General thoracic surgery, Lea and Febiger: Philadelphia; 1989. p. 989-1001.

6. Thomas ML, Anthony AA, Fosh BG, Finch JG, Madden GJ. Oesophageal diverticula. Br J Surg. 2001;88(5):629-42.

7. Castrucci G, Porziella V, Granone PL, Picciocchi A. Tailored surgery for esophageal body diverticula. Eur J Cardiothorac Surg. 1998;
14(4):380-7.

8. Zaninotto G, Portale G, Costantini M, Merigliano S, Guirroli E, Rizzetto $\mathrm{C}$, et al. Long-term outcome of operated and unoperated epiphrenic diverticula. J Gastrointest Surg. 2008;12(9):1485-90. doi: 10.1007/s11605-008-0570-3. Epub 2008 Jul 12.

9. D'Journo XB, Ferraro P, Martin J, Chen LQ, Duranceau A. Lower esophageal sphincter dysfunction is part of the functional abnormality in epiphrenic diverticulum. Br J Surg. 2009;96(8):892900. doi: $10.1002 /$ bjs.6652.

10. Nehra D, Lord RV, DeMeester TR, Theisen J, Peters JH, Crookes PF, et al. Physiologic basis for the treatment ofepiphrenic diverticulum. Ann Surg. 2002;235(3): 346-54.

11. Orringer MB. Epiphrenic diverticula: fact and fable. Ann Thorac Surg. 1993;55(5):1067-8.

12. Altorki NK, Sunagawa M, Skinner DB Thoracic esophageal diverticula. Why is operation necessary? J Thorac Cardiovasc Surg. 1993;105(2):260-4.

13. Thomas RC Carcinoma in epiphrenic diverticula. Ann Thorac Surg. 1968:6(1):85-7.

14. Thomas ML, Anthony AA, Fosh BG, Finch JG, Maddern GJ. Oesophageal diverticula. Br J Surg. 2001;88(5):629-42.

15. Zaninotto G, Portale G, Costantini M, Merigliano S, Guirroli E, Rizzetto C, et al. Long-term outcome of operated and non operated epiphrenic diverticula. J Gastrointest Surg. 2008;12(9):1485-90. doi: 10.1007/s11605-008-0570-3. Epub 2008 Jul 12.

16. Zaninotto G, Portale G, Costantini M, Zanatta L, Salvador R, Ruol A. Therapeutic strategies for epiphrenic diverticula: systematic review. World J Surg. 2011;35(7):1447-53. doi: 10.1007/s00268-011-1065-z.

17. Jordan PH Jr, Kinner BM. New look at epiphrenic diverticula. World J Surg. 1999;23(2):147-52.

18. Klaus A, Hinder RA, Swain J, Achem SR. Management of epiphrenic diverticula. J Gastrointest Surg. 2003;7(7):906-11.

19. Rosati R, Fumagalli U, Bona S, Zago M, Celotti S, Bisagni P, et al. Laparoscopic treatment of epiphrenicdiverticula. J Laparoendosc Adv Surg Tech A. 2001 Dec;11(6):371-5.

20. Del Genio A, Rossetti G, Maffetton V, Renzi A, Brusciano L, Limongelli $P$, et al. Laparoscopic approach in the treatmentof epiphrenic diverticula: long-term results. Surg Endosc. 2004;18(5):741-5.

21. Fernando HC, Luketich JD, Samphire J, Alvelo-Rivera M, Christie NA, Buenaventura PO, et al Minimally-invasive operation for esophageal diverticula. Ann Thorac Surg. 2005; 80(6):2076-80.

22. Peracchia A, Bonavina $L$, Rosati $R$, et al Thoracoscopic resection of epiphrenic diverticula in "Minimally invasive surgery of the foregut" (2001) QMP Inc, St. Louis, p. 110-116.

23. van der Peet DL, Klinkenberg-Knol EC, Berends FJ, Cuesta MA. Epiphrenic diverticula: minimally invasive approach and repair in five patients. Dis Esophagus. 2001;14(1):60-2.

24. Chan DSY, Foliaki A, Lewis WG, Clark GWB, Blackshaw GRJC. Systematic review and metaanalysis of surgical treatment of non-Zenker oesophageal diverticula. J Gastrointest Surg. 2017;21(6): 1067-1075. doi: 10.1007/s11605-017-3368-3. Epub 2017 Jan 20.

25. Hirano Y, Takeuchi H, Oyama T, Saikawa Y, Niihara M, Sako H, et al. Minimally invasive surgery for esophageal epiphrenic diverticula: the results of 133 patients in 25 published series and our experience. Surg Today. 2013;43(1):1-7. doi: 10.1007/s00595-012-0386-3. Epub 2012 Oct 21.

26. Allen TH, Clagett OT. Changing concepts in the surgical management of pulsion diverticula of the lower esophagus.J Thorac Cardiovasc Surg. 1965;50(4):455-62.

27. Campos GM, Vittinghoff E, Rabl C, Takata M, Gadenstätter M, Lin F, et al. Endoscopic and surgical treatment of achalasia: a systematic review and meta-analysis. Ann Surg. 2009;249(1):45-57. doi: 10.1097/SLA.0b013e31818e43ab. 